\title{
Embolizations of Iatrogenic Lower Limb Vascular Injuries after Orthopaedics Surgery
}

\author{
Francesco Giurazza*, Fabio Corvino, Gianluca Cangiano, Errico Cavaglià, Francesco Amodio, Giuseppe De \\ Magistris and Raffaella Niola
}

Department of Interventional Radiology, Italy

*Corresponding author: Francesco Giurazza, Department of Interventional Radiology, AORN Cardarelli di Napoli, Italy.

Received Date: November 16, 2018

Published Date: December 10, 2018

\begin{abstract}
Introduction: This study aims to evaluate safety and efficacy of endovascular embolizations in a series of patients presenting with arterial vascular injuries of the lower limbs following orthopaedic interventions.

Materials and methods: Ten patients have been retrospectively enrolled having pulsatile mass, painful lower limb and anemia (hemoglobin $<7 \mathrm{~g} / \mathrm{dl}$ ).

Result: Vascular lesions were pseudoaneurysm, bleeding with extraluminal contrast agent blush of the terminal arterial segment, laceration and bleeding with extraluminal contrast agent blush of the arterial main trunk. The clinical and procedural technical success was achieved in all cases.

Conclusion: Endovascular embolizations have prooven to be a safe and effective approach to treat arterial vascular lesions occurring after different orthopaedics intervention of the lower limb.
\end{abstract}

\section{Introduction}

Arterial injuries involving lower limbs are rare; main causes are traffic related trauma and iatrogenic lesions during orthopaedics surgical procedures [1]. The clinical manifestations of these injuries typically result in hemorrhagic or ischemic symptoms. Open vascular surgery has been the gold standard in the management of vascular extremity injuries for many years [2] but in the last two decades endovascular techniques have proved to be as effective as open surgery and have been increasingly adopted for treating these lesions [1].

This study focuses on the endovascular embolization treatment of lower limb iatrogenic lesions following orthopaedics surgery. Until today this issue has been discussed in literature mainly in the form of case reports concerning vascular complications of a specific orthopaedic intervention [3-6]; the purpose of this paper is now to evaluate safety and efficacy of endovascular embolization in a series of patients presenting with arterial injuries of the lower limbs following different orthopaedic interventions.

\section{Materials and Methods}

In the last 8 years ten patients have been treated with endovascular arterial embolization for iatrogenic vascular lesions occurring after orthopaedics surgery of the lower limb:
4 males and 6 females, mean age of 66 years ranging from 23 to 90 years. Orthopaedics interventions consisted of: 2 gamma nail positioning after intertrochanteric femoral fracture, 3 cemented total hip arthroplasty, 1 non cemented total hip arthroplasty, 1 anterior cruciate ligament reconstruction, 1 femoral stabilization with metallic plate and screws fixation after displaced diaphysis fracture, 1 stabilization of a tibial lateral condyle fracture and 1 tibial stabilization with Ilizarov fixation after displaced diaphysis fracture. Clinical presentations consisted of palpable pulsatile mass, painful and reduced lower limb motion or visible hematoma; eight patients presented also with anemia (hemoglobin $<7 \mathrm{~g} / \mathrm{dl}$ ).

\section{Result}

Interval time between orthopaedics surgery and embolization ranged between 0 and 67 days (mean: 20 days). Overall twelve vessels were injured; in two patients the vessels involved were two, while in eight cases there was a single vessel injured. The injured arterial vessels were: superficial external pudendal artery (1), inferior gluteal artery (1) (Figure 1), deep femoral artery (1) (Figure 1), lateral circumflex femoral artery (2) (Figure 2), medial circumflex femoral artery (1), articular branch of descending genicular artery (1), perforating femoral arteries (3), posterior tibial recurrent artery (1) and anterior tibial artery (1). 


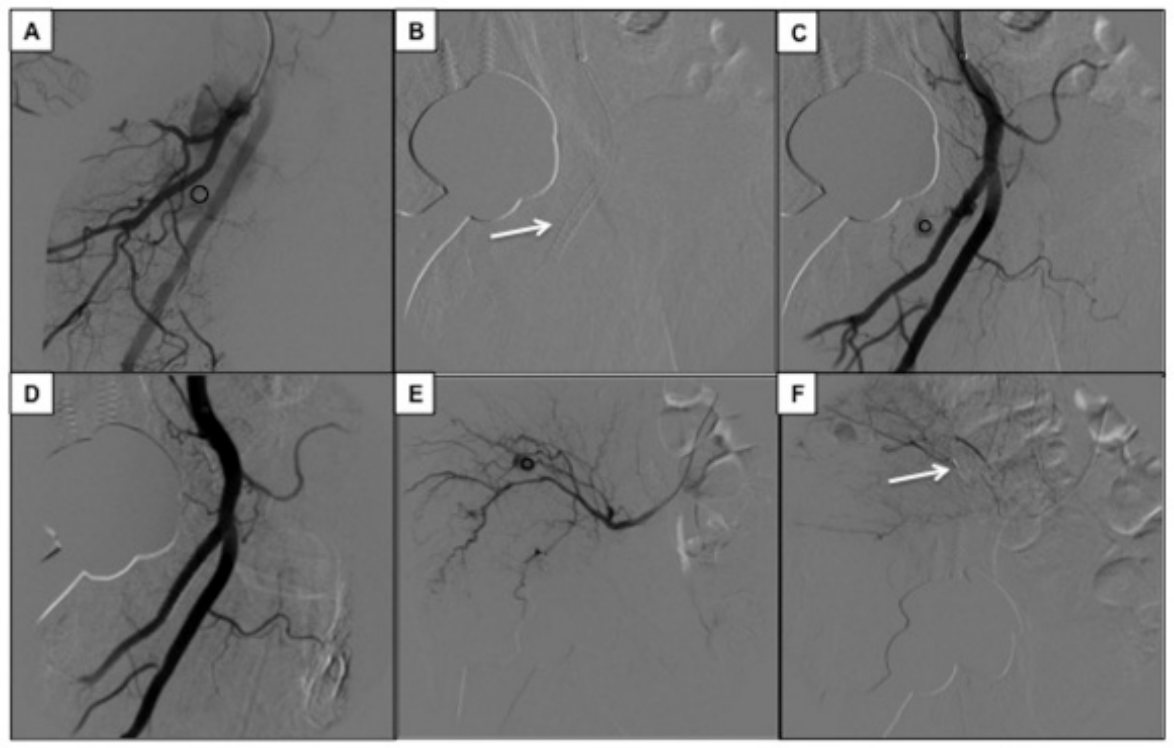

Figure 1: 90 years old woman treated with non cemented total right hip arthroplasty after femoral neck fracture. 12 hours after the intervention she was critically anemic despite transfusions $(\mathrm{Hb}: 5.8 \mathrm{mg} / \mathrm{dl})$ with a wide hematoma of the thigh. CT showed a massive bleeding from the right deep femoral artery whose main trunk was lacerated and a concomitant $11 \mathrm{~mm}$ pseudoaneurysm refurnished from the distal portion of the right inferior gluteal artery was diagnosed. Postero-anterior angiography (A) showing a massive bleeding (black circle) from the main trunk of the right deep femoral artery. Release of the balloon expanded covered stent (white arrow) (B) positioned just after the origin of the vessel in order to preserve the superficial femoral artery patency. Control after the first balloon inflation (C) showing reduction of the extraluminal contrast extravasation with a persistent small amount (black circle). Second control 15 minutes after another balloon inflation (D) showing complete resolution of the bleeding and stent patency. Postero-anterior angiography $(E)$ detecting a $11 \mathrm{~mm}$ pseudoaneurysm of the right inferior gluteal artery successfully treated after embolization with microcoil release (white arrow) (F).

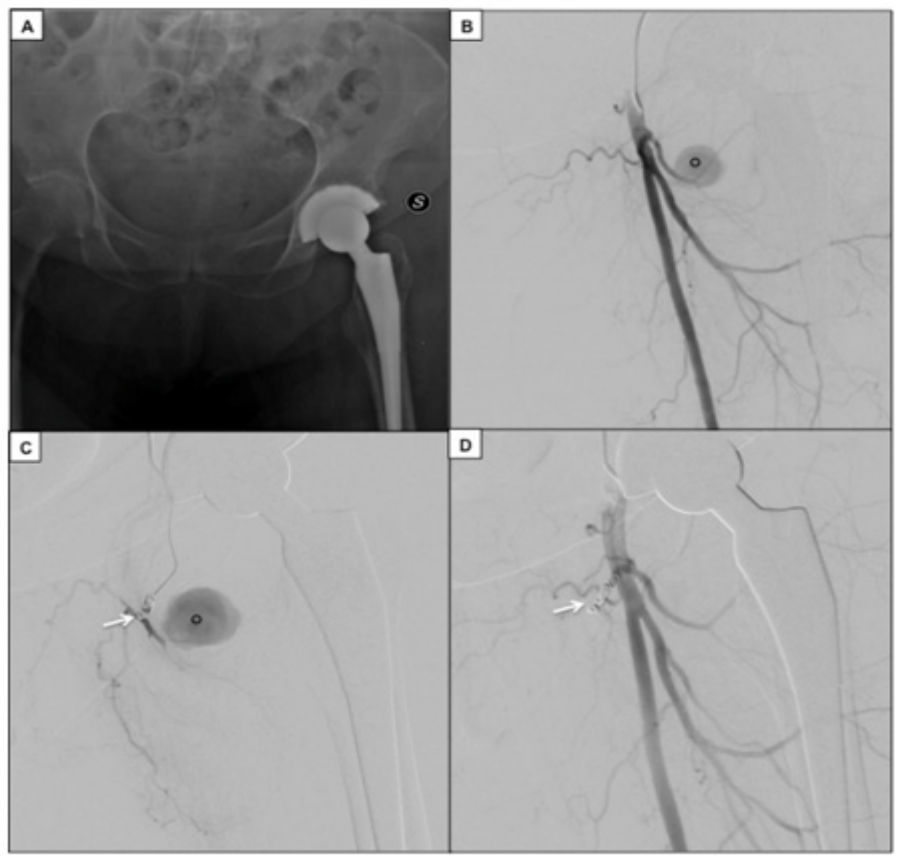

Figure 2: 82 years old woman presenting with a pulsatile mass in the left groin, pain and anemia, 53 days after orthopaedics surgery for left cemented total hip arthroplasty. X-rayshowed prothesis correctly positioned but eco-colordoppler exam demonstrated a pseudoaneurysm in correspondance of the pulsatile mass. Angiography confirmed a $30 \mathrm{~mm}$ pseudoaneurysm of the lateral circumflex femoral artery, successfully treated with microcoils embolization (Concerto ev3®, Irvine CA, USA; MicroNester CookMedical ${ }^{\circledR}$ USA). Postero-anterior X-ray showing cemented total hip arthroplasty correctly in site (A). Diagnostic angiography in postero-anterior projection (B) detecting a pseudoaneurysm of the lateral circumflex femoral artery (black circle); procedural angiography in oblique projection with a 2.7 Fr microcatheter positioned into the left lateral circumflex femoral artery and microcoil release (white arrow) (C); final angiography demonstrating complete exclusion of the pseudoaneurysm after occlusion with microcoils (white arrow) of the left lateral circumflex femoral artery (D).

The typologies of arterial injury were: pseudoaneurysm $58.3 \%$ (7), bleeding with extraluminal contrast agent blush of the terminal arterial segment $25 \%$ (3), laceration and bleeding with extraluminal contrast agent blush of the arterial main trunk $16.6 \%$ (2). The embolizing agents adopted were microcoils $66.7 \%$ (8), glue $8.3 \%$ (1), microplug $8.3 \%$ (1), particles $8.3 \%$ (1), covered 
stent $8.3 \%$ (1). Clinical and technical success was achieved in all cases $(100 \%)$. No major complications related to the embolization procedure were recorded.

\section{Discussion}

Different types of vascular complications have been described in literature after orthopaedics surgery of lower limbs, mainly in form of case reports: vessel laceration, hemorrhage, vascular compression, intimal flap tear, pseudoaneurysm, arteriovenous aneurysm, arterial thromboembolism and ligation [3-6,7], Lesions can be determined by multiple mechanisms: direct acute perforation by an instrument as retractor, screw or scalpel, chronic and slow erosion of a vessel by protruding screws, migration of a loose component, indirect injury resulting from stretching of atherosclerotic or calcified arteries, thermal damage from the polymerisation of cement near to blood vessel [8]. These patients receiving anticoagulants, as subcutaneous heparin, take longer to seal traumatized vessels and are more likely to experience prolonged bleeding. These injuries may be identified intraoperatively, or days to years after the surgery; patients may present with pain, swelling, bleeding, ischemia, or a combination of these symptoms [6].

\section{Conclusion}

In this series endovascular embolizations have prooven to be a safe and effective approach to treat arterial vascular lesions occurring after different orthopaedics intervention of the lower limb; orthopaedic surgeons should be aware of the support that interventional radiologists could provide in case of vascular complications.

\section{Acknowledgement}

None.

\section{Conflict of Interest}

No conflict of interest.

\section{References}

1. Maleux G, Herten PJ, Vaninbroukx J, Thijs M, Nijs S, et al. (2012) Value of percutaneous embolotherapy for the management of traumatic vascular limb injury. Acta Radiol 53(2): 147-152.

2. Hafez HM, Woolgar J, Robbs JV (2001) Lower extremity arterial injury: results of 550 cases and review of risk factors associated with limb loss. J Vasc Surg 33(6): 1212-9.

3. Mello W, De Brito WE, Migon EZ and Borges A (2011) Pseudoaneurysm of the medial inferior genicular artery after anterior cruciate ligament reconstruction. Arthroscopy 27(3): 442-445.

4. Sadat U, Naik J, Verma P, See TC, Cousins C, et al. (2008) Endovascular management of pseudoaneurysms following lower limb orthopedic surgery. Am J Orthop 37(5): E99-E102.

5. Rachakonda A, Qato K, Khaddash T, Carroccio A, Pamoukian V, et al. (2015) Ultrasound-Guided Thrombin Injection of Genicular Artery Pseudoaneurysm. Ann Vasc Surg 29(5): 1017.e11-1017.e13.

6. Huynh S, Kayssi A, Koo K, Rajan D, Safir O, et al. (2016) Avulsion injury to the profunda femoris artery after total hip arthroplasty. J Vasc Surg 64(2): 494-496.

7. Barquet A, Gelink A, Giannoudis PV (2015) Proximal femoral fractures and vascular injuries in adults: Incidence, aetiology and outcomes. Injury 46(12):2297-2313.

8. Alshameeri Z, Bajekal R, Varty K, Khanduja V (2015) Iatrogenic vascular injuries during arthroplasty of the hip. Bone Joint J 97-B: 1447-55. 